\title{
Glycerol-A Viable Solvent for Absorption of Highly Polar Solutes I: Behaviour of Molecular Interactions
}

\author{
Jacques J. Scheepers and Edison Muzenda
}

\begin{abstract}
This work focused on the potential of glycerol as a scrubbing solvent for stripping of highly polar volatile organic compounds (VOCs) from industrial waste gas streams. Results for glycerol interactions were compared to those of water. Solubility predictions in the form of infinite dilution activity coefficients were made using the Modified UNIFAC Dortmund group contribution model, which was set up on a Microsoft Excel spreadsheet specifically designed for this purpose. Activity coefficients for VOCs in water and glycerol (highly polar solvents) increased with an increase in the length of the VOC solute nonpolar tail. Furthermore, activity coefficients decreased with an increase in the degree of branching of the VOC solute. The results show that low chain length highly polar alcohol, ketone, organic acid and aldehyde solutes are very soluble in glycerol. Diols, dicarbonyl aldehydes, some ketone derivatives and the ethanolamines also show good solubility. Highly polar diesters and ester derivatives are less soluble in glycerol and water. This study has found glycerol to be a better scrubbing solvent than water in treating highly polar VOCs. However it is possible that the standard method of building up the glycerol molecule in the UNIFAC may result in under-predictions of activity coefficients and thus special group interaction parameters for glycerol are required.
\end{abstract}

Index Terms-Activity coefficients, glycerol, solubility, UNIFAC, van der Waals.

\section{INTRODUCTION}

The rapid depletion of world oil reserves is a real threat facing industry today, especially for the petrochemical and transportation industries. The transportation sector has addressed this threat by intensifying research into alternative fuels sources. Biodiesel, produced mainly from the transesterification of vegetable oils, has been identified as a viable fuel source and is used extensively to supplement mineral diesel stocks. However the rapid rise in biodiesel production has resulted in an oversupply of the byproduct glycerol into world markets, thereby depressing glycerol prices to the point where glycerol is in some cases even cheaper than water [1]. Whilst $\mathrm{Gu}$ and Jérôme [1] have described some potential uses of glycerol as a solvent, it is important to explore other applications.

Chemical industries are facing increasing pressure due to environmental legislation to limit (or preferably eliminate) the emission of volatile organic compounds (VOCs) into the atmosphere. In order to curb their VOC emissions, many

Manuscript received March 31, 2014; June 3, 2014. This work was financially supported by the University of Johannesburg.

The authors are with the Department of Chemical Engineering, Faculty of Engineering and the Built Environment, University of Johannesburg, Johannesburg, South Africa (tel: 0027-11-5596817; fax: 0027-11-5596430; e-mail: jacquesjscheepers@gmail.com, emuzenda@uj.ac.za). chemical industries are using end-of-pipe technologies to remove VOCs from waste gas streams. Since physical absorption processes are proven technologies for treatment of industrial end-of-pipe emissions, the use of 'green' solvents as scrubbing agents has been investigated, especially because many of the available scrubbing solvents are themselves also VOCs. Since most VOCs saturate quickly in water, the use of organic solvents as scrubbing agents has been investigated. Intensive preliminary investigation has already shown that esters, especially those comprising biodiesel, are a viable scrubbing solvent for removing nonpolar to moderately polar VOCs from waste gas streams [2]-[12]. However ester solvents are incapable of stripping highly polar VOCs from waste gas streams. Being a trihydric (triol) alcohol, glycerol is a highly polar protic solvent with a high boiling point (thus possessing low volatility) and is biodegradable [1]. Whilst this indicates that nonpolar to moderately polar solutes will be insoluble in glycerol, highly polar VOCs should be fairly soluble in glycerol.

Because VOCs are usually present in very dilute concentrations in waste gas streams [13], it is usually impractical and uneconomical to perform phase equilibrium measurements during the preliminary design phase. For this reason prediction of thermodynamic data such as infinite dilution activity coefficients using a suitable thermodynamic model such as the UNIFAC group contribution method is required. For the purposes of this study, preliminary studies on the feasibility of glycerol as a suitable scrubbing solvent was conducted using the Modified UNIFAC Dortmund group contribution model, which was developed in 1987 by Weidlich and Gmehling [14]. The computations were performed using a Microsoft Excel spread sheet specifically designed for this purpose.

\section{THE MODIFIED UNIFAC DORTMUND METHOD}

The Modified UNIFAC Dortmund group contribution method for calculating activity coefficients $(\gamma)$ is the sum of a combinatorial contribution which describes the excess Gibbs energy arising due to differences in molecular size and shape, and a residual $(R)$ contribution which describes the excess Gibbs energy differences due to molecular interactions [15], i.e.

$$
\ln \gamma_{i}=\ln \gamma_{i}^{C}+\ln \gamma_{i}^{R}
$$

The combinatorial term $\left(\ln \gamma_{i}^{C}\right)$ is described as [14]

$$
\ln \gamma_{i}^{C}=\ln \Phi_{i}^{\prime}+1-\Phi_{i}^{\prime}-5 q_{i}\left(\ln \frac{\Phi_{i}}{\theta_{i}}+1-\frac{\Phi_{i}}{\theta_{i}}\right)
$$


where the volume (or segment) fraction parameter $\left(\Phi_{i}\right)$ and modified volume fraction parameter $\left(\Phi_{i}^{\prime}\right)$ of component $(i)$ is defined as [14]

$$
\begin{gathered}
\Phi_{i}=\frac{r_{i} x_{i}}{\sum_{j} r_{j} x_{j}} \\
\Phi_{i}^{\prime}=\frac{r_{i}^{3 / 4}}{\sum_{j} r_{j}^{3 / 4} x_{j}}
\end{gathered}
$$

The van der Waals area fraction $\left(\theta_{i}\right)$ is calculated as [14]

$$
\theta_{i}=\frac{q_{i} x_{i}}{\sum_{j} q_{j} x_{j}}
$$

The pure-component volume parameter $\left(r_{i}\right)$ and the pure-component area parameter $\left(q_{i}\right)$ are defined as [16]

$$
\begin{aligned}
r_{i} & =\sum_{k} v_{k}^{(i)} R_{k} \\
q_{i} & =\sum_{k} v_{k}^{(i)} Q_{k}
\end{aligned}
$$

where parameter $\left(v_{k}^{(i)}\right)$ is the number of groups of kind $(k)$ in a molecule of component $(i)$ and is thus always an integer. Values for the group area parameters $\left(Q_{k}\right)$ and the group volume parameters $\left(R_{k}\right)$ are obtained from published tables. The residual term $\left(\ln \gamma_{i}^{R}\right)$ remains unchanged from the Original UNIFAC and is expressed as follows [16]:

$$
\ln \gamma_{i}^{R}=\sum_{k} v_{k}^{(i)}\left[\ln \Gamma_{k}-\ln \Gamma_{k}^{(i)}\right]
$$

Parameter $\left(\Gamma_{k}\right)$ is the residual activity coefficient of the group $(k)$, whereas parameter $\left(\Gamma_{k}^{(i)}\right)$ represents the residual activity coefficient of group $(k)$ in a reference solution which contains only molecules of type $(i)$. The residual activity coefficients $\left(\Gamma_{k}\right)$ and $\left(\Gamma_{k}^{(i)}\right)$ are both computed using (9) [16]:

$$
\ln \Gamma_{k}=Q_{k}\left\{1-\left(\ln \sum_{m} \Theta_{m} \Psi_{m k}\right)-\sum_{m}\left(\frac{\Theta_{m} \Psi_{k m}}{\sum_{n} \Theta_{n} \Psi_{n m}}\right)\right\}
$$

Parameter $\left(\Theta_{m}\right)$, the surface area fraction for group $m$, is described as [16]

$$
\Theta_{m}=\frac{Q_{m} X_{m}}{\sum_{n} Q_{n} X_{n}}
$$

where $\left(X_{m}\right)$, the group mole fraction for group $(m)$, is determined as follows [15]:

$$
X_{m}=\frac{\sum_{j} v_{m}^{(j)} X_{j}}{\sum_{j} \sum_{n} v_{n}^{(j)} X_{j}}
$$

The modified group interaction parameter $\left(\psi_{m n}\right)$ which, unlike that of the Original UNIFAC was made to be temperature dependent, is expressed as follows [14]:

$$
\Psi_{m n}=\exp \left[-\frac{a_{m n}+b_{m n} T+c_{m n} T^{2}}{T}\right]
$$

\section{The NATURE OF VOC SOlUTE - GlyCEROL SOLVENT INTERACTIONS}

Infinite dilution activity coefficients were predicted for 30 highly polar VOCs in glycerol. The results obtained were compared to the VOC interactions with water, which is also a highly polar solvent used as a conventional absorbent solvent for polar solutes. A mole fraction of $1 \times 10^{-5}$ was selected to represent infinite dilution conditions, as recommended by Alessi et al [13]. Activity coefficients for interactions between VOCs and water were measured at $30^{\circ} \mathrm{C}$, considered a standard operating temperature for many absorber systems [2]. For interactions between glycerol and VOCs, activity coefficients were measured at $60^{\circ} \mathrm{C}$ because at lower temperatures glycerol is probably too viscous for effective mass transfer to occur between solute and solvent in absorption columns [1].

\section{A. Interactions with Increasing Solute Chain Length}

The alcohol family was studied to ascertain the effect of glycol interactions with solutes increasing in hydrocarbon chain length, as shown in Fig. 1. The interaction of water with pentanol $(5 \mathrm{C}-\mathrm{OH})$ is not shown but gave an infinite dilution activity coefficient of 129.2 .

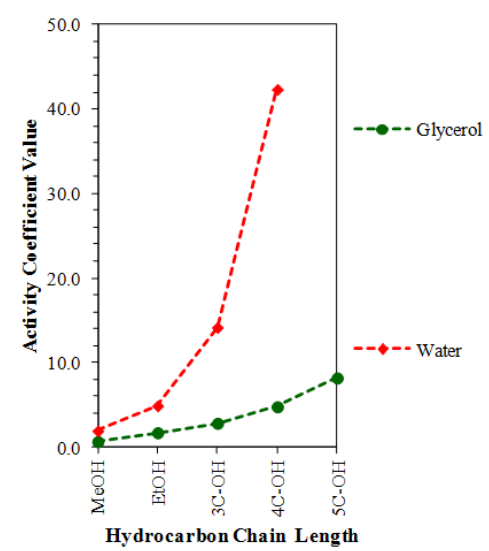

Fig.1 Interactions of straight-chain alcohols with glycerol and water

It is evident that solubility of the VOC solute decreases with an increase in the solute hydrocarbon chain length, because the solute becomes increasingly nonpolar with increasing chain length. This is attributed to the strength of the hydrogen bonds at the site of the hydroxyl $(\mathrm{OH})$ group being 
increasingly shielded with increasing nonpolar chain length. The increasing length of the nonpolar hydrocarbon tails (where only London forces dominate) interfere with the solutes accessing the hydrogen bonding sites of the glycerol and water molecules. Thus solute-solute interactions (being much weaker London interactions) are incapable of supplying enough solvation energy to facilitate the breaking of the high energy solvent-solvent interactions break for solute-solvent bonding to take place. It is clear from Fig. 1 that solubility in water is highly influenced by solute chain length compared to glycerol. This may be due to glycerol having more active sites for hydrogen bonding to occur between solute and solvent.

\section{B. The Effect of Branching on Solute-Solvent Interactions}

The interaction of glycerol with $\mathrm{C} 4$ alcohol isomers was studied to determine the effect of branching on VOC solubility, Fig. 2.

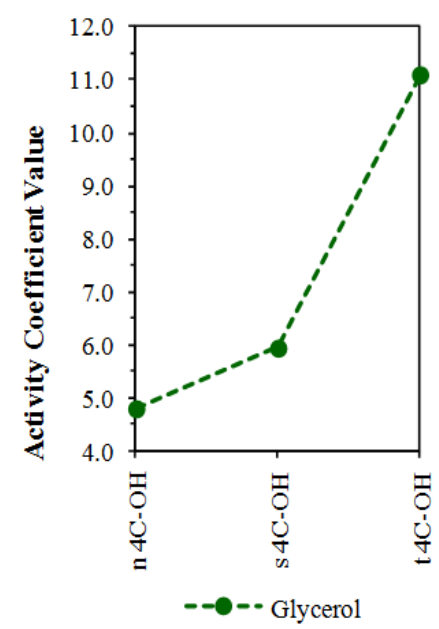

Fig. 2. Effects on $\mathrm{C} 4$ alcohol branching on interactions with glycerol

VOC solubility decreased with an increase in the degree of branching of the VOC solute. As for an increase in hydrocarbon chain length, branching serves to shield the solute hydroxyl site, thereby limiting its contact with the hydroxyl sites of the glycerol solvent. Thus Keesom (dipole-dipole) interaction between the solute and solvent molecules is hindered resulting in the increase in activity coefficients experienced.

\section{GLYCEROL INTERACTION WITH VARIOUS VOC FAMILIES}

\section{A. Alcohols}

The interaction of the solvents, glycerol and water with short-chained methanol and the diols ethylene glycol and propylene glycol is shown in Fig. 3. It can be seen from Fig. 3 that methanol $(\mathrm{MeOH})$ and the diol ethylene glycol, being highly polar, are very soluble in both water and glycerol. Naturally propylene glycol, having a nonpolar tail, is not as soluble in glycerol and is fairly insoluble in water, hence the high activity coefficient for the water/ propylene glycol interaction.

\section{B. Aldehydes and Dicarbonyl Aldehydes}

The interaction of short-chain aldehydes and dicarbonyl aldehydes with water and glycerol is shown in Fig. 4.

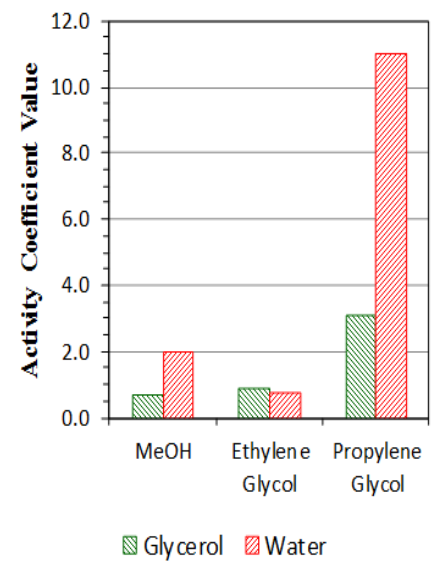

Fig. 3. Interaction of water and glycerol with alcohols

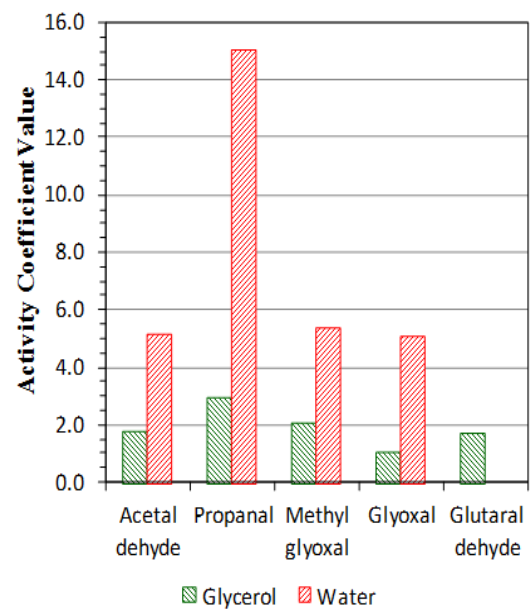

Fig. 4. Interaction of water and glycerol with aldehydes

Aldehydes do not possess a hydrogen atom bonded to a highly electronegative atom such as oxygen in their makeup; hence aldehydes are not hydrogen bond (H-bond) donors. However due to the high electronegativity of their carbonyl oxygen they are H-bond acceptors, thus allowing them to participate in intermolecular bonding with molecules such as water and glycerol, which are themselves H-bond donors. Thus aldehydes are in general only moderately polar. The dicarbonyl aldehydes are highly polar because of the high dipole moment created by two carbonyl oxygen atoms, thus dicarbonyl atoms are quite soluble in glycerol, and to a much lesser extent in water.

Glyoxal, the shortest-chained dicarbonyl aldehyde, possessed the lowest activity coefficient because the lack of any nonpolar tail combined with the two electronegative oxygen atoms makes glyoxal extremely polar. This allows glyoxal to participate readily in Keesom interactions with the solvents. The activity coefficient of glutaraldehyde in water not shown in Fig. 4 is 44.15 because glutaraldehyde is a C5 dicarbonyl aldehyde with the electronegative centers positioned relatively far apart from each other. It is unclear why glutaraldehyde is still quite soluble in glycerol. The large decrease in solubility from acetaldehyde to propanal is attributed to the increase in length of the nonpolar hydrocarbon tail.

\section{Ketones and Ketone Derivatives}

The interaction of short-chained acetone and ketone derivatives with glycerol and water is shown in Fig. 5. 


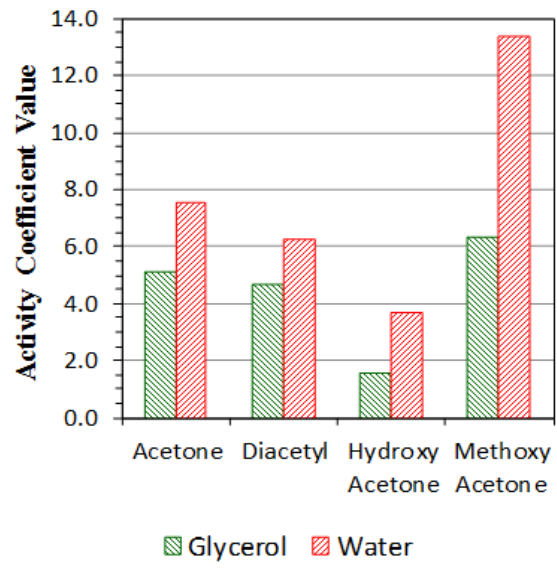

Fig. 5. Interaction of water and glycerol with ketones

Like aldehydes, the ketones studied are only H-bond acceptors due to the carbonyl oxygen, with the exception of hydroxy acetone. Hydroxy acetone contains a hydroxyl group and is thus also an H-bond donor, hence the highest solubility in glycerol and water. Solubility of ketones in glycerol and water is generally lower than that of aldehydes possibly because of the nonpolar hydrocarbon groups flanking both sides of the ketone carbonyl interfering with ketone access to the hydrogen bonding sites of the solvents. It is also possible that, since ketones possess a greater dipole moment than their functional isomer aldehydes, it requires more energy to break solute-solute interactions before solute-solvent bonding can take place. This would explain the behaviour of glycerol's interaction with propanal and its function isomer acetone. It however does not explain why interactions between propanal and water yield much higher activity coefficients than those between acetone and water. In this instance it is possible that the length of the hydrocarbon tail of propanal being longer on one side than that of acetone creates interference with access to the bonding sites of the water molecules.

\section{Organic Acids}

The interaction of organic acids with glycerol and water is shown in Fig. 6. The infinite dilution activity coefficient of formic acid in glycerol is not shown as it could not be modeled using UNIFAC due to the lack of group interactions parameters for formic acid interaction with hydroxyl groups). A very low activity coefficient value is however expected for the interaction between glycerol and formic acid.

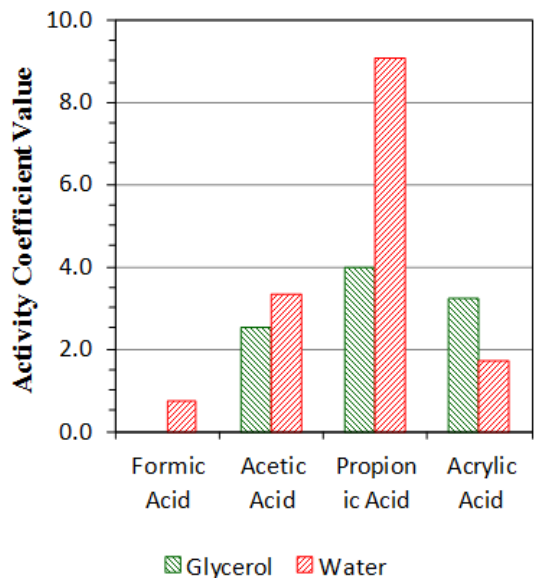

Fig. 6. Interaction of water and glycerol with organic acids
It is evident from Fig. 6 that the lower chain length organic acids are very soluble in glycerol and water. This is attributed to the fact that organic acids possess two hydrogen bonding sites - a carbonyl group as an H-bond acceptor site and a hydroxyl group as an H-Bond donor site. Naturally H-bond donor sites can also act as H-bond acceptor sites. As expected, solubility decreases with an increase in the length of the nonpolar tail.

\section{E. Ethanolamines}

The interaction between ethanolamines in glycerol and water is shown in Fig. 7.

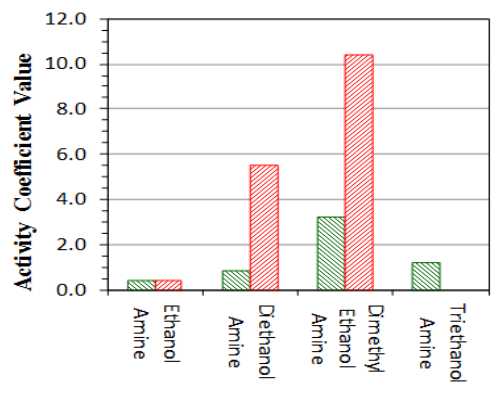

$\mathbb{Q}$ Glycerol Water

Fig. 7. Interaction of water and glycerol with ethanolamines

It was found that activity coefficients increased from ethanol amine, to diethanolamine, to triethanolamine with the addition of each hydroxyl group to the amine molecule. Thus solubility decreased with an increase in the polarity of the solute molecule, since more energy was required to break solute-solute bonds before solute-solvent bonding could occur. Since triethanolamine (like glycerol) is a triol with three primary hydroxyl groups, it is much more polar than water, hence the extremely high activity coefficient (39.478) for interaction between water and triethanolamine. Since glycerol is a triol with three active H-bond donor sites, diethanolamine and triethanolamine was expected to be more soluble in this solvent than in water, hence the much lower activity coefficients obtained for glycerol-ethanolamine interactions.

It is evident from Fig. 7 that dimethylethanolamine interaction with glycerol yielded the highest activity coefficient of the four solutes studied. This is explained by the interference of the two methyl groups flanking the ethanolamine chain.

\section{F. Highly Polar Diesters and Ester Derivatives}

The results for interactions between diesters, carbonates and lactates with glycerol and water are shown in Table I.

TABLE I: INTERACTION OF WATER AND GLYCEROL WITH DIESTERS \& ESTER DERIVATIVES

\begin{tabular}{lccccc}
\hline \hline & \multicolumn{5}{c}{ Activity Coefficients - Ester Solute/ Solvent } \\
Interactions \\
\cline { 2 - 7 } & & & & & \\
Solvent & & & & & \\
Glycerol & 56.391 & 14.557 & 22.263 & 5.018 & 7.859 \\
Water & 894.04 & 205.52 & 50.132 & 16.988 & 46.423 \\
\hline \hline
\end{tabular}


It is clear from Table I that, even if highly polar, esters are generally less soluble in both water and glycerol. This is because the lengthy nonpolar hydrocarbon tails flank both sides of esters effectively shielding the polar sites reducing interaction with the highly polar solvents. The lactates, possessing a hydroxyl group, are the most soluble in both solvents.

\section{CONCLUSION AND RECOMMENDATIONS}

Glycerol is a more effective solvent than water in absorbing highly polar solutes from industrial waste gas streams. Diols such as ethylene glycol, triethylene glycol and tetraethylene glycol have to be treated as special groups in UNIFAC modeling since the standard method of building up these molecules in UNIFAC results in under-prediction of activity coefficients for binary interactions. It is therefore possible that triols such as glycerol may have to be treated in a similar fashion. It is thus recommended that experimental work on the interactions modeled in this work be carried out to evaluate the accuracy of the predictions.

\section{ACKNOWLEDGMENT}

The authors are very grateful to the University of Johannesburg's Faculty of Engineering and the Built Environment Research Committee and First National Battery, SA for their continued multi-faceted support.

\section{REFERENCES}

[1] Y. Gu and F. Jérôme, "Glycerol as a sustainable solvent for green chemistry," Green Chem., vol. 12, no. 7, pp. 1127-1138, June. 2010.

[2] K. Bay, H. Wanko, and J. Ulrich, "Absorption of volatile organic compounds in biodiesel: determination of infinite dilution activity coefficients by Headspace Gas Chromatography," Chem. Eng. Res. Des., vol. 84, no. A1, pp. 22-27, Jan. 2006.

[3] K. Bay, H. Wanko, and J. Ulrich, "Biodiesel - hoch siedendes absorbens für die gasreinigung," Chemie Ingenieur Technik, vol. 76, no. 3, pp. 328-333, Mar. 2004.

[4] J. J. Scheepers, E. Muzenda, and M. Belaid, "Influence of temperature and molecular structure on organics-biodiesel interactions using group contribution methods," in Proc. the 2012 International Conference of Manufacturing Engineering and Engineering Management, World Congress on Engineering (IAENG), 2012, vol. 3, pp. 1411-1416.

[5] J. J. Scheepers, E. Muzenda, and M. Belaid, "Influence of structure on fatty acid ester-alkane interactions," in Proc. International Conference on Chemical Engineering and its Applications (ICCEA'2012), Bangkok, 2012, pp. 93-102.

[6] J. J. Scheepers and E. Muzenda, “Alkenes - ester polymeric solvents thermodynamic interactions - Part 1," in Proc. International Conference on Ecology, Agriculture and Chemical Engineering, 2012, pp. 229-232.

[7] J. J. Scheepers, E. Muzenda, and M. Belaid, "Alkenes - ester polymeric solvents thermodynamic interactions - Part 2," in Proc. International conference on Nanotechnology and Chemical Engineering, Bangkok, 2012, pp. 54-57.

[8] J. J. Scheepers, E. Muzenda, and M. Belaid, "Intermolecular dynamics between aromatic compounds and ester polymeric solvents," in Proc. International Conference of Planetary Scientific Research Centre, 2013, pp. 37-41.

[9] J. J. Scheepers and E. Muzenda, The Solubility of Volatile Organic Compounds in Biodiesel, Saarbrücken, Germany: LAP Lambert Academic Publishing, Aug. 2013.

[10] J. J. Scheepers and E. Muzenda, "Temperature dependence of limiting activity coefficients in c18 ester solvents - Part 1," in Proc. IIE Int'l Conference on Emerging Trends in Engineering and Technology (ICETET'2013), 2013, pp. 155-159.
[11] J. J. Scheepers and E. Muzenda, "Temperature dependence of limiting activity coefficients in c18 ester solvents - Part 2," in Proc. IIE Int'l Conference on Emerging Trends in Engineering and Technology (ICETET' 2013), 2013, pp. 160-164.

[12] J. J. Scheepers and E. Muzenda, "Influence of temperature on volatile organic compounds - Ester solvents interactions," South African Journal of Chemical Engineering.

[13] P. Alessi, M. Fermeglia, and I. Kikic, "Significance of dilute regions," Fluid Phase Equilib., vol. 70, no. 2-3, pp. 239-250, Dec. 1991.

[14] U. Weidlich and J. Gmehling, "A modified UNIFAC model. 1. Prediction of VLE, hE, and $\gamma^{\infty}$," Ind. Eng. Chem. Res., vol. 26, no. 7, pp. 1372-1381, Jul. 1987.

[15] A. Fredenslund, J. Gmehling, M. L. Michelson, P. Rasmussen, and J. M. Prausnitz, "Computerised design of multicomponent distillation columns using the UNIFAC group contribution method," Ind. Eng. Chem. Process Des. Dev., vol. 16, no. 4, pp. 450-462, Apr. 1977.

[16] A. Fredenslund, R. L. Jones, and J. M. Prausnitz, "Group-contribution estimation of activity coefficients in nonideal liquid mixtures," AlChE J., vol. 21, no. 6, pp. 1086-1099, November 1975.

[17] A. Fredenslund, J. Gmehling, and P. Rasmussen, Vapor-Liquid Equilibria Using Unifac - A Group Contribution Method, Elsevier Science, Amsterdam, Netherlands, 1977.

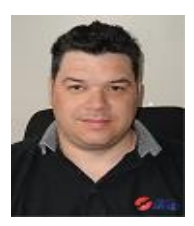

Jacques J. Scheepers holds a Master's degree (MTech) in Chemical Engineering, obtained in 2014 at the University of Johannesburg, South Africa.

He currently works for First National Battery in Benoni, South Africa, as a technical support engineer. He has also worked previously for an EPCm company, Foster Wheeler (SA) based in Midrand, South Africa, as a project engineer. Prior to this, he worked as a process engineer for Sasol Wax, South Africa. Jacques has co-authored numerous conference papers and one journal article on the absorption of volatile organic compounds using ester solvents. Together with Edison Muzenda, Jacques has also published a book entitled "The Solubility of Volatile Organic Compounds in Biodiesel" (Saarbrücken, Germany: LAP Lambert Academic Publishing, 2013). His current research interests remain the search of suitable solvents for the absorption of volatile organic compounds.

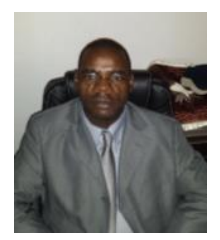

Edison Muzenda is a full professor of chemical engineering, the research and postgraduate coordinator as well as the head of the Environmental and Process Systems Engineering Research Group in the Department of Chemical Engineering at the University of Johannesburg. Professor Muzenda holds a BSc Hons degree (ZIM, 1994) and a $\mathrm{PhD}$ degree in chemical engineering (Birmingham, 2000). He has more than 15 years' experience in academia which he gained at different Institutions: National University of Science and Technology, University of Birmingham, Bulawayo Polytechnic, University of Witwatersrand, University of South Africa and the University of Johannesburg. Through his academic preparation and career, Edison has held several management and leadership positions such as member of the student representative council, research group leader, university committees' member, staff qualification coordinator as well as research and postgraduate coordinator. Edison's teaching interests and experience are in unit operations, multi-stage separation processes, environmental engineering, chemical engineering thermodynamics, entrepreneurship skills, professional engineering skills, research methodology as well as process economics, management and optimization. He is a recipient of several awards and scholarships for academic excellence. His research interests are in green energy engineering, integrated waste management, volatile organic compounds abatement and as well as phase equilibrium measurement and computation. He has published more than 200 international peer reviewed and refereed scientific articles in journals, conferences and books. Edison has supervised 28 postgraduate students, 4 postdoctoral fellows as well as more than 140 Honours and BTech research students. He serves as reviewer for a number of reputable international conferences and journals. Edison is a member of the Faculty of Engineering and Built Environment Research and Process, Energy and Environmental Technology Committees. He has also chaired several sessions at International Conferences. Edison is an associate member of the Institution of Chemical Engineers (AMIChemE), member of the International Association of Engineers (IAENG); associate member of Water Institute of Southern Africa (WISA), Associate Editor for the South African Journal of Chemical Engineering as well as a member of the Scientific Technical Committees and Editorial Boards of several scientific organization. 